\title{
Intragastric bile acid concentrations are unrelated to symptoms of flatulent dyspepsia in patients with and without gallbladder disease and postcholecystectomy
}

\author{
R G P WATSON AND A H G LOVE \\ From the Department of Medicine, The Queen's University of Belfast, Belfast, N Ireland
}

\begin{abstract}
SUMmARY It has been proposed that duodenogastric reflux may be the basic underlying mechanism which gives rise to symptoms of flatulent dyspepsia. Fasting and postprandial gastric juice bile acid concentrations were measured in patients with flatulent dyspepsia with and without gall bladder disease and postcholecystectomy. There were 13 patients with gall bladder disease, 12 with normal gall bladders and 13 postcholecystectomy. Gastric juice was obtained by intubation. Bile acid concentrations were compared with 21 controls and 15 asymptomatic subjects with gall bladder disease. For 21 patients with gall bladder disease who underwent cholecystectomy, levels were again assessed postoperatively to allow correlation with outcome. The occurrence of reflux and the resultant gastric juice bile acids did not correlate with symptoms. Concentrations postcholecystectomy, including asymptomatic subjects were significantly higher than controls $(\mathrm{p}<0.01)$. It is concluded that limited duodenogastric reflux is common and need not be associated with symptoms even when the resultant intra-gastric concentrations are higher than normal.
\end{abstract}

There is a great interest in the clinical significance of duodenogastric reflux. ${ }^{\prime}$ It has been proposed by Johnson $^{23}$ that this may be the basic mechanism responsible for producing flatulent dyspepsia, including fatty food intolerance. These symptoms have traditionally been regarded as symptoms of gall bladder disease, but there is now considerable evidence that such an association is likely to be coincidental. Symptoms commonly occur in patients with no evidence of gall bladder disease, ${ }^{4-8}$ and it is also apparent that cholecystectomy often fails to relieve flatulent dyspepsia..$^{211}$

It, therefore, suggests that symptoms could be due to a mechanism other than gall bladder disease and is common to all patients with the same symptoms. Using a radiological technique Johnson ${ }^{23}$ showed that duodenogastric reflux commonly occurred in dyspeptic patients with and without gall bladder disease. Reflux was regarded as a functional disturb-

\footnotetext{
Address for correspondence: Dr R G P Watson, Department of Medicine, The Queen's University of Belfast, Institute of Clinical Science, Grosvenor Road, Belfast, BT12 6BJ.

Received for publication 13 May 1986
}

ance which directly gives rise to symptoms but might also be expected to predispose to symptomatic 'bile gastritis' as envisaged by Capper $e$ t al. ${ }^{12}$

The aim of this study was to investigate whether intragastric bile acid concentrations are related to symptoms of flatulent dyspepsia in patients with and without gall bladder disease and postcholecystectomy. The presence of bile acid in the stomach is an indication that duodenogastric reflux has taken place and because bile acids have been shown to be damaging to gastric mucosa ${ }^{1314}$ their concentration may indicate the potential cytotoxic effect of reflux.

\section{Methods}

SUBJECTS

There were five groups of subjects:-

(1) Patients with gall bladder disease and dyspepsia (D). There were 13 subjects, two men, 11 women, mean age 40.6 years. They were recruited from surgical waiting lists for cholecystectomy. The indications for surgery as stated by the surgeon was dyspepsia in 10 cases, and in three they were placed in this group when questioning revealed a significant 
history of dyspepsia in addition to previous acute cholecystitis $^{2}$ or biliary colic. ${ }^{1}$ All had demonstrable gall bladder disease by means of cholecystography and/or ultrasonography. Cholecystectomy was subsequently done in 11 patients and chronic cholecystitis was confirmed in all.

Dyspepsia was defined as the presence of one or more of five principal symptoms. These were flatulence (feelings of abdominal wind or belching), upper abdominal fullness including inability to finish a normal sized meal, epigastric discomfort after food, other than the pain of acute cholecystitis or biliary colic, nausea and/or vomiting and fatty food intolerance. A questionnaire was used to assess the duration frequency and severity of symptoms. Severity was adjudged to be mild, moderate, or severe in the same way as Price: ${ }^{4}$ mild if a doctor had not been consulted and no remedy tried, moderate if some action had been taken but the symptoms had not prevented normal activities and severe if normal activity had been upset. A symptom was counted as present if the history was at least three months and it was moderate or severe. Mild symptoms were only included if they occurred every day. The mean number of symptoms per patient was 3.4. All had flatulence or fullness and 12 fatty food intolerance. Co-existent peptic ulcer disease was excluded by endoscopy or barium meal examination. Patients with prominent bowel symptoms, indicating possible irritable bowel syndrome, were also excluded.

(2) Patients with gall bladder disease and no dyspepsia (non-D): There were 15 patients in this group, four men, 11 women, mean age 53.5 years. Thirteen had a history of previous acute cholecystitis or bilary colic and were recruited from surgical waiting lists for elective cholecystectomy. There was no significant history of dyspepsia either before or after their acute illness. Two subjects had 'silent gall stones' detected as incidental findings. Gall bladder disease was demonstrated by cholecystography and/or ultrasonography. When cholecystectomy was subsequently done in 13 cases chronic cholecystitis was confirmed in all.

(3) Subjects with $x$-ray negative and endoscopy negative dyspepsia (x-R-ve). There were 12 in the group, four men, eight women, mean age 43.5 years. Symptoms were similar to D, all had flatulence or fullness, except one who had a significant degree of fatty food intolerance and vomiting. The mean number of symptoms per patient was $4 \cdot 3$. All patients had radiological and/or endoscopic investigation to exclude upper gastrointestinal pathology. Gall bladder disease had been excluded by ultrasonography or cholecystography.

(4) Postcholecystectomy subjects with symptoms of dyspepsia (PC): There were 13 patients, two men,
11 women, mean age 52.4 years. In eight cases precholecystectomy symptoms had continued after operation and in five, arisen after a symptom free period. Symptoms were similar to $D$, all had flatulence or fullness except one who had a significant degree of fatty food intolerance and vomiting. The mean number of symptoms per patient was $3 \cdot 4$.

(5) Control subjects (C): There were 21 with a similar sex and age distribution to the other groups, six men 15 women, mean age 45.5 years. None had dyspeptic symtoms and 'silent gall stones' were excluded by ultrasonography.

Subjects were intubated with a size 14 Salem sump nasogastric tube after an overnight fast. The tip of the tube was positioned under direct radiographic screening so that it lay in the gastric antrum. The tube was left in situ for the next 45-60 minutes to allow the patient to settle. The stomach was then completely emptied. For the next $\mathbf{3 0}$ minutes, gastric juice was continuously aspirated. This was the fasting basal sample. A liquid test meal consisting of $18 \mathrm{~g}$ corn oil and $12 \mathrm{~g}$ glucose, made up to $100 \mathrm{ml}$ with warm water was passed down the tube. Samples of juice $(10 \mathrm{ml}$ each) were then taken off at 20 and 40 minutes after the meal and the stomach again completely emptied 60 minutes after the meal. Volumes of each sample and $\mathrm{pH}$ were measured before storage at $-20^{\circ} \mathrm{C}$. When thawed, samples were assayed by an enzymatic technique using $3 \alpha$ hydroxysteroid dehydrogenase as described by Fausa and Skallhegg ${ }^{15}$ and validated for measurement of bile acids in gastric juice by Collins $e t$ al. ${ }^{16}$ The minimum concentration of bile acid which could be reliably detected was $20 \mu \mathrm{mol} / \mathrm{l}$.

For 21 subjects who underwent cholecystectomy $(10 \mathrm{D}, 11$ non-D) the protocol was repeated after operation after an interval of between 2-7 months, mean 4.7. Symptoms were again assessed by questionnaire and they were asked to say whether there had been improvement, no change or worsening.

\section{Results}

The fasting levels of intragastric bile acid were similar fòr all five groups. The distributions were shewed towards zero with concentrations tending to be higher for the postcholecystectomy group but not significantly different when compared using the Mann-Whitney U-test (Fig. 1). The median levels were $21.86 \mu \mathrm{mol} / \mathrm{l}$ (C), 0 (D), 59 (non-D), 27 (x-R-ve) and 225 (PC). The pH of the gastric juice specimens were also not significantly different. The median levels were 1.57 (C), 2.3 (D), 2.5 (non-D), 1.64 (x-R-ve) and 1.68 (PC).

Detectable bile acid and, hence, reflux was present in all groups. The percentage of subjects per groups 


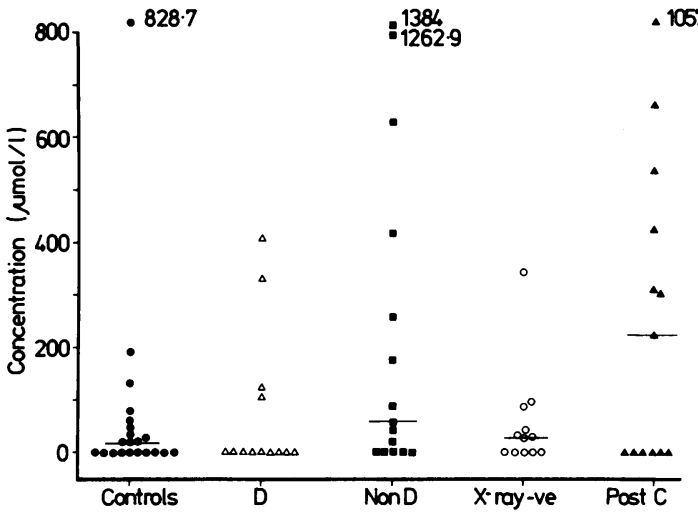

Fig. 1 Fasting bile acid concentrations in gastric juice of controls $(\mathrm{C})$, patients with gall bladder disease and dyspepsia (D), patients with gall bladder disease and no dyspepsia (non-D), patients with ' $x$-ray negative' dyspepsia (x-R-ve) and patients with dyspepsia postcholecystectomy (PC).

in whom reflux occurred was $52 \cdot 4 \%$ (C), $30 \cdot 8 \%$ (D), $66.67 \%$ (non-D), 58.34\% (x-R-ve) and 53.8 (PC). There were no significant differences between the groups as assessed by $\chi^{2}$ analysis.

The mean postprandial concentration for each subject was obtained from the levels at 20,40 , and 60 minutes. In the symptomatic postcholecystectomy group (PC), concentrations were significantly higher than $C, D$, and $x-R$-ve $(p<0.05)$. The non-dyspeptic group with gall bladder disease (non-D) was also significantly higher than $D$ and $x-R$-ve $(p<0.05)$ but not different from PC and controls (Fig. 2). The mean $\mathrm{pH}$ of the postprandial specimens were not significantly different. Median levels were 2.27 (C)

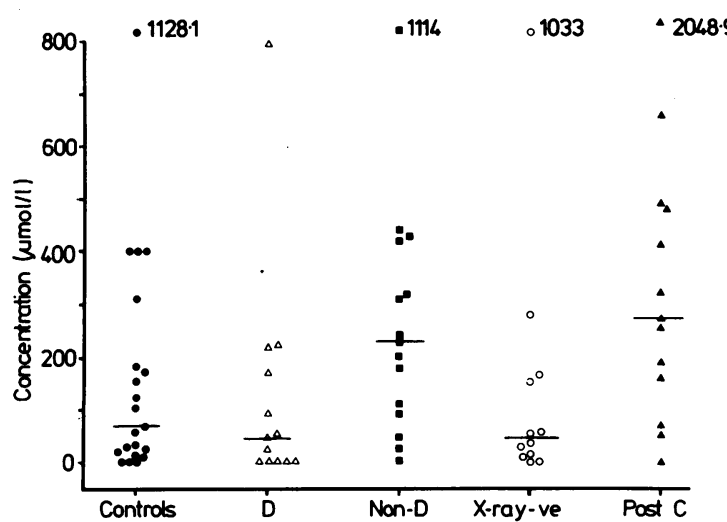

Fig. 2 Mean post-prandial bile acid concentrations in controls $(C)$, patients with gall bladder disease and dyspepsia (D), patients with gall bladder disease and no dyspepsia (non-D), patients with ' $X$-ray negative' dyspepsia $(x-R$-ve) and patients with dyspepsia postcholecystectomy $(P C)$.

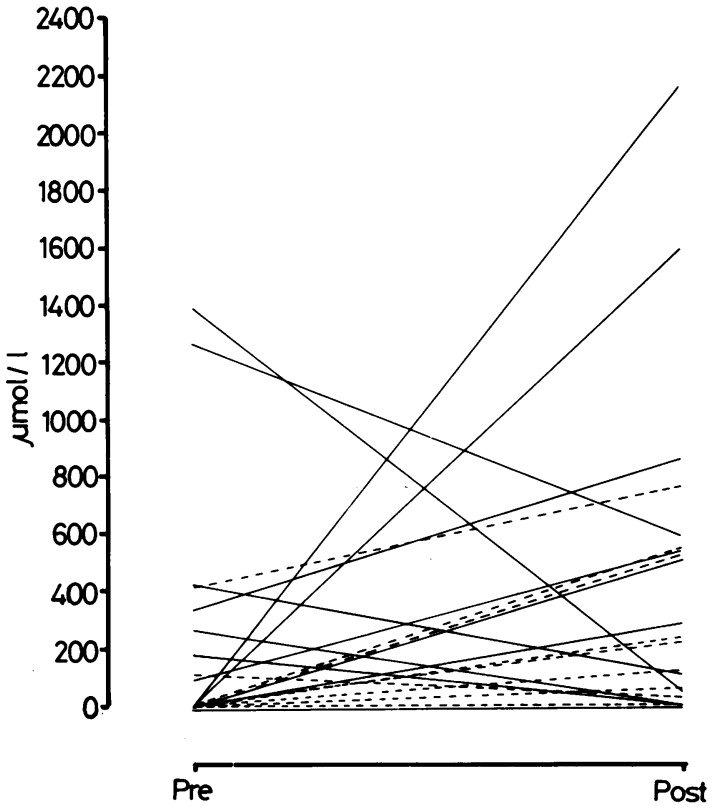

Fig. 3 Comparisons of fasting bile acid concentrations in gastric juice of 21 patients pre-and postcholecystectomy. Dashed lines are nine patients in whom dyspepsia improved after operation.

$2 \cdot 14$ (D), $2 \cdot 42$ (non-D), 1.62 (x-R-ve) and 1.61 (PC). Bile acids were detectable in at least one postprandial sample in the following percentages $85.7 \%$ (C), $61.5 \%$ (D), $93.3 \%$ (non-D), $83.3 \%$ (x-R-ve) and $92.3 \%$ (PC). Only one patient experienced typical symptoms during the test.

For the 21 subjects in whom samples were obtained before and after cholecystectomy, fasting concentrations tended to be higher after surgery than before (Fig. 3). Overall there was no significant difference assessed by the Wilcoxon's sign rank test. Concentrations were higher in 13 patients, less in six and the same in two. For nine patients with dyspepsia who had complete relief of symptoms (three) or definite improvement (six) there was a significant increase in bile acid concentrations $(\mathrm{p}<0 \cdot 05)$. The $\mathrm{pH}$ levels, pre- and postcholecystectomy did not differ significantly. The median $\mathrm{pH}$ precholecystectomy was 2.33 and postcholecystectomy 1.79 .

The mean postprandial concentrations also tended to be higher, but not significantly so (Fig. 4). There was an increase in 12, and a decrease in nine. In the nine improved patients there was again a significant increase in concentrations $(p<0.05)$. The mean postprandial $\mathrm{pH}$ 's did not differe significantly between pre- and postcholecystectomy specimens. The median $\mathrm{pH}$ precholecystectomy was 2.45 and postcholecystectomy $2 \cdot 18$. 


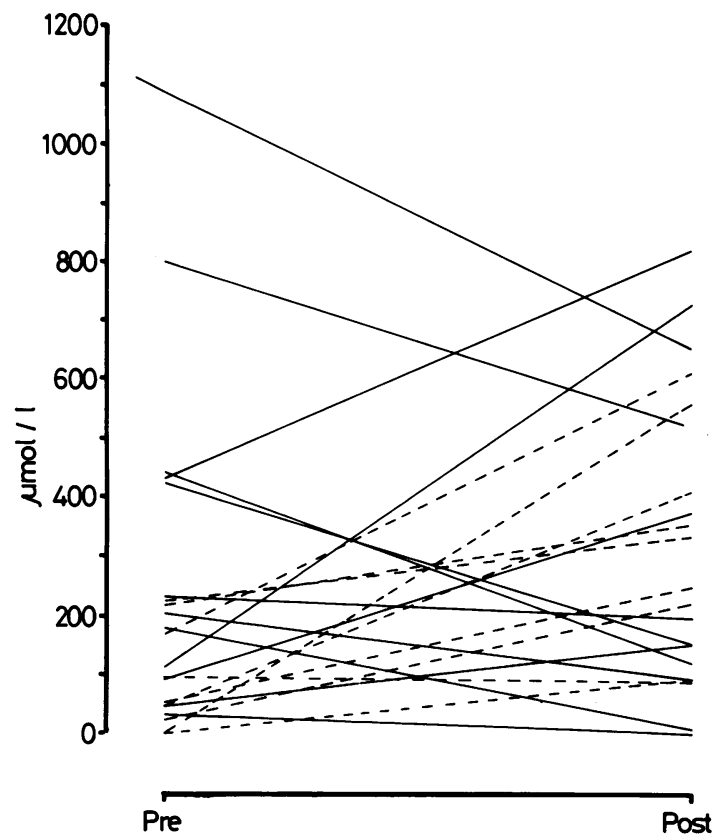

Fig. 4 Comparisons of mean postprandial bile acid concentrations in gastric juice of 21 patients pre-and postcholecystectomy. Dashed lines are nine patients in whom dyspepsia improved after operation.

After operation there were 13 asymptomatic subjects including three who had previously had dyspepsia. Their fasting and mean postprandial concentrations did not differ significantly from the eight subjects who were symptomatic after operation and were also similar to the original symptomatic postcholecystectomy group (PC). In the asymptomatic group fasting levels were greater than controls $(p<0.01)$, postprandial levels did not differ. For the total number of postcholecystectomy subjects studied $(n=34)$ both the fasting and mean postprandial levels were significantly higher than controls $(\mathrm{p}<0.01)$ (Fig. 5).

\section{Discussion}

It is clear from the results that reflux is common in all groups including normal controls and is not specifically associated with symptoms. It might be argued that the use of an intubation technique in the present study is 'invasive' and, thereby, may spuriously provoke reflux. A number of studies which have assessed the degree of reflux associated with intubation have found this to be minimal, ${ }^{17-19}$ however, and intubation is considered to be a valid technique.

The present results of detectable reflux in normal controls is in agreement with other recent studies which have also demonstrated reflux in controls using various techniques - direct sampling ${ }^{20}$ transpyloric and intragastric intubation, ${ }^{21}$ hepatobiliary tracer and gamma camera ${ }^{22}$ and real time ultrasound..$^{23}$ It appears that limited duodenogastric reflux is a normal physiological event and in a small number of cases relatively high levels of bile acid can be detected. In keeping with this only one dyspeptic patient with detectable reflux in the present study experienced typical symptoms.

In terms of the concentrations of bile acid in gastric juice there is no tendency for dyspeptic patients with or without gall bladder disease (D and $x-R-v e$ ) to have higher levels than controls or non-dyspeptic patients with gall-bladder disease (non-D). Indeed, the latter has higher mean postprandial concentrations than $\mathrm{D}$ and $\mathrm{x}-\mathrm{R}$-ve groups. There is similarly no significant difference in concentrations between symptomatic and asymptomatic subjects after cholecystectomy, but levels are higher than controls. It is notable that in nine symptomatic patients with gall-bladder disease, who improved with operation, there was a significant rise in bile acid concentration

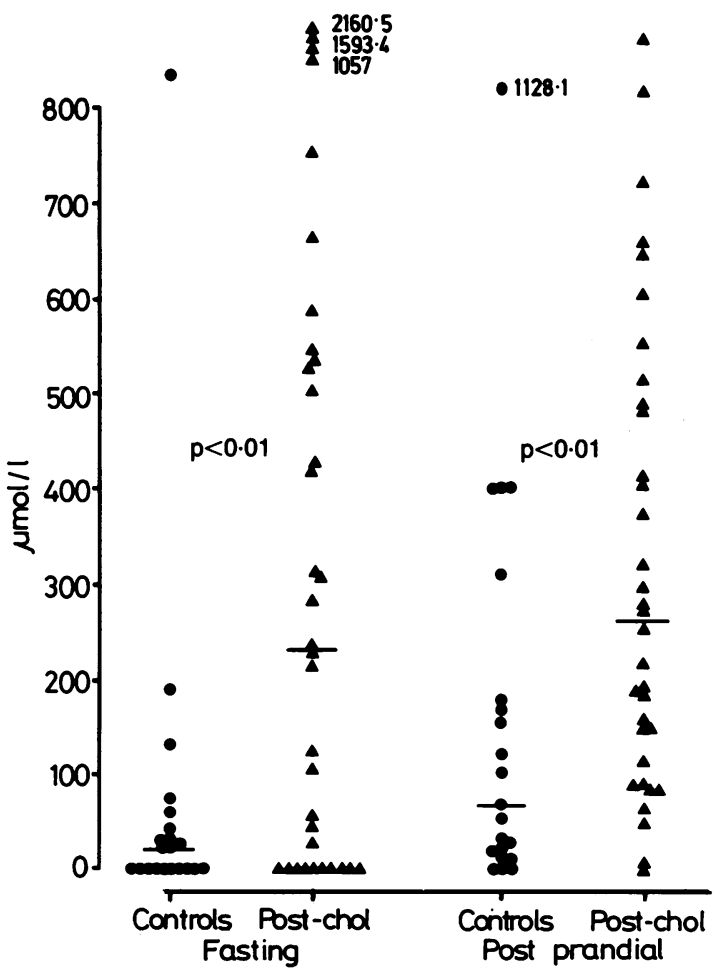

Fig. 5 Fasting and mean postprandial bile acid concentrations in gastric juice of controls and all postcholecystectomy subject $(n=34)$. Median levels shown by horizontal bars. 
after surgery compared with levels taken before. This is contrary to what would be expected if symptoms were simply related to reflux.

The higher bile acid concentrations in gastric juice after cholecystectomy is consistent with previous reports. Brough et al ${ }^{24-26}$ assessed duodenogastric reflux in pre- and postcholecystectomy dogs and man by measuring bilirubin in gastric juice, and the fraction of a hepatobiliary tracer which could be recovered from the stomach. Both were increased after cholecystectomy and they concluded that duodenogastric reflux is increased. This may be correct but it is not the only possible explanation. Bile secretion is continuous after cholecystectomy and bile acid output increased. ${ }^{27}$ This factor alone might predispose an increase of intragastric bile constituents, including bile acids, without any change in duodenogastric reflux. It could also be speculated that the higher level of bile acids after operation could be brought about by a change in bile acid constituents which are more soluble at acid $\mathrm{pH}$, or there could be a rise in gastric juice $\mathrm{pH}$ which would allow more of a given amount of bile acid to remain in solution. In the present study gastric juice $\mathrm{pH}$ changes can be excluded as there was no significant difference in measured $\mathrm{pH}$ between pre- and postcholecystectomy subjects.

Buxbaum ${ }^{28}$ observed from his endoscopic practice that over $50 \%$ if cases of bile gastritis occurred in patients who had previously undergone cholecystectomy. Similarly, other studies ${ }^{29}{ }^{30}$ have associated previous cholecystectomy with the development of bile gastritis. These suggest that at least some postcholecystectomy patients have problems related to duodenogastric reflux. The term 'bile gastritis' has tended to focus attention on bile as the principal damaging agent but it seems likely that other components of refluxed fluid, such as pancreatic juice, may also be important. ${ }^{3132}$ In the case of bile, Harman et $a l^{33}$ have suggested that it may be the composition of bile acids rather than the absolute amount which is important in determining whether gastric problems occur. Either of these factors could account for differences in symptoms among patients with similar levels of total bile acids as found in this study. It is also likely that there are individual patient susceptibilities in injury by the damaging agent or agents.

We, therefore, conclude that limited duodenogastric reflux is probably physiological. The occurrence of reflux and gastric juice concentrations of bile acids in our patients did not correlate with symptoms. It is still possible, however, that duodenagastric reflux is implicated in some cases.

We wish to thank Sister E Crawford for her skilled technical assistance. R G P Watson was supported by a grant from the Northern Ireland Department of Health and Social Services.

\section{References}

1 Clémençon GH, ed. Proceedings of the 2nd International symposium on duodenogastric reflux. Scand $J$ Gastroenterol 1984; 19: suppl. 92.

2 Johnson AG. Pyloric function and gall-stone dyspepsia. Br J Surg 1972; 59: 449-54.

3 Johnson AG. Cholecystectomy and gallstone dyspepsia. Clinical and physiological study of a symptom complex. Ann R Coll Surg Engl 1975; 56: 69-80.

4 Price WH. Gall-bladder dyspepsia. Br Med J 1963; 2: 138-41.

5 Bainton D, Davies GT, Evans UT, Gravelle IH. Gallbladder disease prevalence in a South Wales industrial town. N Engl J Med 1976; 294: 1147-9.

6 Koch JP, Donaldson RM. A survey of food intolerances in hospitalised patients. $N$ Engl J Med 1964; 271: 657-60.

7 Hinkel CL, Muller GA. Correlation of symptoms, age, sex and habitus with cholecystographic findings in 1000 consecutive examinations. Gastroenterology 1957; 32: 807-15.

8 Earlam RJ, Thomas M. The clinical significance of gallstones and their radiological investigation. Br J Surg 1978; 65: 164-7.

9 Bodvall B. The postcholecystectomy syndromes. In: Bouchier IAD, ed. Clinics in gastroenterology disease of the biliary tract. London: WB Saunders, 1973; 2: 103-26.

10 Bockus HL. Gastroenterology 3rd ed, vol 3. London: WB Saunders, 1976: 767.

11 Bates T, Mercer JC, Harrison M. Symptomatic gallstone disease: before and after cholecystectomy. [Abstract] Gut 1984; 25: A579.

12 Capper WM, Butler TJ, Kilby JO, Gibson MJ. Gall stones, gastric secretion and flatulent dyspepsia. Lancet 1967; 1: 413-5.

13 Davenport HW. Destruction of the gastric mucosal barrier by detergents and urea. Gastroenterology 1968; 54: 175-81.

14 Ritchie WP, Shearburn III EW. Acute gastric mucosal ulcerogenesis is dependent on the concentration of bile salt. Surgery 1976; 80: 98-105.

15 Fausa O, Skalhegg BA. Quantitative determination of bile acids and their conjugates using thin-layer chromatography and a purified 3-hydroxysteroid dehydrogenase. Scand J Gastroenterol 1974; 9: 249-54.

16 Collins BJ, Watt PCH, O'Reilly T, McFarland RJ, Love AHG. Measurement of total bile acids in gastric juice. $J$ Clin Pathol 1984; 37: 313-6.

17 Hollinger A, Ammann R, Sialer G. Pelliha R. Quantitative assessment of duodenogastric reflux after vagotomy with or without pyloroplasty - a clinical investigation. Scand J Gastroenterol 1981; 16: suppl. 67: 55-7.

18 Muller-Lissner SA, Fimmel CJ, Will N. Muller-Duysing W, Heinzel F, Blum AL. Effect of gastric and transpyloric tubes on gastric emptying and duodenogastric reflux. Gastroenterology 1982; 83: 1276-9. 
19 Wolverson RL, Sorgi M, Mosimann F, Donovan IA, Harding LK, Alexander-Williams J. Does gastric entubation cause entero-gastric reflux? Scand $J$ Gastroenterol 1984; 19: suppl. 92: 41-3.

20 Keane FB, Dimango EP, Malagelada J-R. Duodenogastric reflux in humans: Its relationship to fasting antroduodenal motility and gastric, pancreatic and biliary secretion. Gastroenterology 1981; 81: 726-31.

21 Muller-Lisner SA, Fimmel CJ, Sonnenberg A, et al. Novel approach to quantify duodenogastric reflux in healthy volunteers and in patients with type 1 gastric ulcer. Gut 1983; 24: 510-8.

22 Sonnenberg A, Muller-Lissner SA, Weiser MF, MullerDuysing W. Heinzel F, Blum AL. Effect of liquid meals on duodenogastric reflux in humans. Am J Physiol 1982; 243: G42-G47.

23 King PM, Adam RD, Pryde A, McDicken WN, Heading RC. Relationships of human antroduodenal motility and transpyloric fluid movement: non-invasive observations with real-time ultrasound. Gut 1984; 25: 1384-91.

24 Brough WA, Taylor TV, Torrance HB. Role of the pylorus and gall bladder in reflux associated gastritis. [Abstract] Gut 1984; 25: A578-A9.

25 Brough WA, Taylor TV, Torrance HB. The effect of cholecystectomy on duodenogastric reflux in dogs and humans. Scand J Gastroenterol 1984; 19: suppl. 92: 242-4.

26 Brough WA, Taylor TV, Torrance HB. The effect of cholecystectomy on duodenogastric reflux in patients with previous peptic ulcer surgery. Scand $J$ Gastroenterol 1984; 19: suppl. 92: 255-6.

27 Malagelada J-R, Go VLW; Summershill WHJ, Gamble WS. Bile acid secretion and biliary bile acid composition altered by cholecystectomy. Dig Dis 1973; 18: 455-9.

28 Buxbaum KL. Bile gastritis occurring after cholecystectomy. Am J Gastroenterol 1982; 77: 305-11.

29 Warshaw AL. Bile gastritis without prior gastric surgery: contributing role of cholecystectomy. Am J Surg 1979; 137: 527-1.

30 Kalima T, Sjoberg J. Bile reflux after cholecystectomy. Scand J Gastroenterol 1981; 16: suppl. 67: 153-6.

31 Lawson $\mathrm{HH}$. Effect of duodenal contents on the gastric mucosa under experimental conditions. Lancet 1964; 1: 469-72.

32 Delaney JP, Brodie TA, Robbins PL. Pyloric reflux gastritis: the offending agent. Surgery 1975; 77: 764-72.

33 Harman JW. Duong T, Gaducz TR. Bile acids are not equally damaging to the gastric mucosa. Surgery 1978; 84: 79-86. 